\title{
GEOFFROY DEROME
}

\section{Transcendance des valeurs des fonctions automorphes sur $\mathfrak{H} \times \mathfrak{H}$}

Journal de Théorie des Nombres de Bordeaux, tome 15, $\mathrm{n}^{\circ} 3$ (2003), p. 683-696

<http://www.numdam.org/item?id=JTNB_2003_15_3_683_0>

(C) Université Bordeaux 1, 2003, tous droits réservés.

L'accès aux archives de la revue «Journal de Théorie des Nombres de Bordeaux » (http://jtnb.cedram.org/) implique l'accord avec les conditions générales d'utilisation (http://www.numdam.org/conditions). Toute utilisation commerciale ou impression systématique est constitutive d'une infraction pénale. Toute copie ou impression de ce fichier doit contenir la présente mention de copyright.

\section{Numdam}

Article numérisé dans le cadre du programme

Numérisation de documents anciens mathématiques

http://www.numdam.org/ 


\title{
Transcendance des valeurs des fonctions automorphes sur $\mathfrak{H} \times \mathfrak{H}$
}

\author{
par GEOFFROY DEROME
}

\begin{abstract}
RÉSUMÉ. Soit $\Gamma$ un groupe arithmétique agissant proprement discontinument sur $\mathfrak{H} \times \mathfrak{H}$ de covolume fini. On sait que l'espace $\Gamma \backslash \mathfrak{H} \times \mathfrak{H}$ est isomorphe à l'ensemble des points complexes d'une variété algébrique quasi-projective $V_{\Gamma}$ définie sur $\overline{\mathbb{Q}}$. Soit $J_{\Gamma}$ : $\mathfrak{H} \times \mathfrak{H} \rightarrow V_{\Gamma}(\mathbb{C})$ une application holomorphe invariante par l'action de $\Gamma$ et correctement normalisée. Grâce au résultats obtenus par P. Cohen, H. Shiga et J. Wolfart, on sait que $J_{\Gamma}\left(z_{1}, z_{2}\right) \notin V_{\Gamma}(\overline{\mathbb{Q}})$ si $z=\left(z_{1}, z_{2}\right)$ est un point algébrique non spécial de $\mathfrak{H} \times \mathfrak{H}$. Dans cet article, nous allons montrer que nous avons $J_{\Gamma}\left(z_{1}, z_{2}\right) \notin V_{\Gamma}(\overline{\mathbb{Q}})$ si $z_{1}$ et $z_{2}$ sont deux éléments de $\mathfrak{H}$, l'un étant algébrique et l'autre transcendant.
\end{abstract}

ABSTRACT. Let $\Gamma$ be an arithmetic group acting properly discontinuously on the product of two copies of the poincaré upper space $\mathfrak{H} \times \mathfrak{H}$ with finite covolume. One knows that the space $\Gamma \backslash \mathfrak{H} \times \mathfrak{H}$ is isomorphic to the set of complex points of a quasi-projective variety $V_{\Gamma}$ defined over $\overline{\mathbb{Q}}$. Let $J_{\Gamma}: \mathfrak{H} \times \mathfrak{H} \rightarrow V_{\Gamma}(\mathbb{C})$ be an holomorphic mapping invariant under $\Gamma$ and properly normalized. Thanks to P. Cohen, H. Shiga and J. Wolfart's results, one knows that $J_{\Gamma}\left(z_{1}, z_{2}\right) \notin V_{\Gamma}(\overline{\mathbb{Q}})$ if $z=\left(z_{1}, z_{2}\right)$ is an algebraic non special point of $\mathfrak{H} \times \mathfrak{H}$. In the present article, we shall show, that we have $J_{\Gamma}\left(z_{1}, z_{2}\right) \notin V_{\Gamma}(\overline{\mathbb{Q}})$ if $z_{1}$ and $z_{2}$ are two elements of $\mathfrak{H}$, one of which is algebraic, the other transcendental.

\section{Introduction}

Soit $G$ un groupe algébrique réductif connexe défini sur $\mathbb{Q}$ tel que la partie abélienne de $G(\mathbb{R})$ soit compacte. Désignons par $G(\mathbb{R})^{+}$la composante connexe de l'identité de $G(\mathbb{R})$ et par $K$ un sous-groupe compact maximal de $G(\mathbb{R})^{+}$. Supposons que le quotient $G(\mathbb{R})^{+} / K$ possède une structure complexe invariante et par conséquent soit isomorphe en tant que variété analytique complexe à un domaine symétrique borné $\mathfrak{D} \subset \mathbb{C}^{m}$ pour un certain entier $m \geq 1$. On dit qu'un point $z \in \mathfrak{D}$ est spécial s'il est point fixe

Manuscrit reçu le 19 novembre 2001. 
d'un tore maximal $T \subset G$ défini sur $\mathbb{Q}$ pour lequel $T(\mathbb{R})$ est compact. Supposons que $\mathfrak{D}$ soit réalisé dans $\mathbb{C}^{m}$ de telle manière que les points spéciaux appartiennent à $\mathfrak{D} \cap \overline{\mathbb{Q}}^{m}$. Si $\Gamma$ est un sous-groupe arithmétique de $G$, il existe une application $\Gamma$-invariante holomorphe $J=J(\mathfrak{D}, \Gamma)$ de $\mathfrak{D}$ à valeurs dans $\mathbb{P}_{\mathbb{C}}^{N}$ induisant un isomorphisme entre $\Gamma \backslash \mathfrak{D}$ et l'ensemble des points complexes d'une variété algébrique quasi-projective $V_{\Gamma}$. De plus, Faltings [5] a prouvé que la variété algébrique $V_{\Gamma}$ était définie sur $\overline{\mathbb{Q}}$ et que de plus $V_{\Gamma}$ était bien défini à $\bar{Q}$-isomorphisme près en imposant que tous les points spéciaux $z \in \mathfrak{D}$ aient pour image un point algébrique de $V_{\Gamma}$ par $J$. On appelle un tel triplet $\left(\mathfrak{D}, J, V_{\Gamma}\right)$ un modèle normalisé sur $\overline{\mathbb{Q}}$ pour $(G, \Gamma)$.

Dans [1], Paula Cohen fait la conjecture suivante :

Conjecture 1.1. Soit $\left(\mathfrak{D}, J, V_{\Gamma}\right)$ un modèle normalisé sur $\overline{\mathbb{Q}}$ pour $(G, \Gamma)$ avec $\mathfrak{D} \subset \mathbb{C}^{m}$, alors $z \in \mathfrak{D} \cap \overline{\mathbb{Q}}^{m}$ et $J(z) \in V_{\Gamma}(\overline{\mathbb{Q}})$ si et seulement si $z$ est un point spécial.

Dans ce même article $P$. Cohen démontre que la conjecture 1.1 est vraie dans le cas particulier suivant :

Théorème 1.2 (P. Cohen-H. Shiga-J. Wolfart). Soit ( $\left.\mathfrak{D}, J, V_{\Gamma}\right)$ un modèle normalisé sur $\overline{\mathbb{Q}}$ pour $(G, \Gamma)$ pour lequel $(G, \mathfrak{D})$ admet un plongement symplectique, alors $z \in \mathfrak{D} \cap \overline{\mathbb{Q}}^{m}$ et $J(z) \in V_{\Gamma}(\overline{\mathbb{Q}})$ si et seulement si $z$ est un point spécial.

Références. On peut consulter l'article de [1] ou celui de [7]. En effet, dans ce cas, $\mathfrak{D}$ est un espace de paramètre pour des familles analytiques de variétés abéliennes polarisées d'un certain type. D'autre part, un élément d'une famille est définie sur $\overline{\mathbb{Q}}$ si et seulement si cet élément est représenté par $z \in \mathfrak{D}$ vérifiant $J(z) \in V_{\Gamma}(\overline{\mathbb{Q}})$. Ces remarques ont permis aux auteurs d'utiliser avec profit le théorème du sous-groupe analytique de Wüstholz.

Dans le présent article, nous nous plaçons dans le cas particulier où le groupe réductif $G$ est tel que $\mathfrak{D}=G(\mathbb{R})^{+} / K$ s'identifie à $\mathfrak{H} \times \mathfrak{H}$.

Nous faisons la conjecture suivante :

Conjecture 1.3. Les hypothèses étant celles de la conjecture 1.1, si $\mathfrak{D}$ s'identifie à $\mathfrak{H} \times \mathfrak{H}$, alors étant donné $z=\left(z_{1}, z_{2}\right)$ non spécial tel que au moins un des deux nombres $z_{1}$ ou $z_{2}$ soit algébrique, on a $J(z) \notin V_{\Gamma}(\overline{\mathbb{Q}})$.

Le but du présent article est de montrer le résultat suivant :

Théorème 1.4. La conjecture 1.3 est vraie dans le cas particulier où $(G, \mathfrak{D})$ possède un plongement symplectique.

D'après le théorème 1.2 , on sait déjà qu'étant donnés $z_{1}$ et $z_{2}$ deux nombres algébriques appartenant au demi-plan supérieur de Poincaré, si 
$z=\left(z_{1}, z_{2}\right)$ n'est pas un point spécial alors $J_{\Gamma}(z) \notin V_{\Gamma}(\overline{\mathbb{Q}})$. Ici, nous allons montrer qu'il suffit en fait qu'un seul des deux nombres $z_{1}$ ou $z_{2}$ soit algébrique pour avoir le résultat escompté.

Remarque. Dans le cas particulier où $G=\mathrm{Sl}_{2, F}$ avec $F$ un corps totalement réel de degré 2 , le domaine $\mathfrak{D}$ s'identifie à $\mathfrak{H} \times \mathfrak{H}$, et on trouve dans [1] (voir aussi [2] et [7]) une preuve du fait que $J_{\Gamma}(z) \notin V_{\Gamma}(\overline{\mathbb{Q}})$ si $z=\left(z_{1}, z_{2}\right)$ avec $z_{i} \in \mathfrak{H}, i=1,2$ n'est pas un point spécial et si au moins un des $z_{i}$ est algébrique. Ici, nous allons nous intéresser à d'autres groupes réductifs $G$.

Le plan de cet article est le suivant. Dans le premier paragraphe on pose les notations et on effectue quelques rappels. Dans le second paragraphe on montrera que les variétés abéliennes associées à $z=\left(z_{1}, z_{2}\right)$ avec $z_{i} \in \mathfrak{H}$ pour $i=1$ et 2 , l'un étant transcendant, l'autre algébrique sont simples. Dans le troisième paragraphe, nous rappellerons un résultat de l'article de [3] qui ne s'applique que pour les variétés abéliennes simples et qui s'appuie sur le théorème du sous-groupe analytique. Le dernier paragraphe est consacré aux applications et résultats finaux.

\section{Familles de variétés abéliennes polarisées de type PEL.}

Nous allons dans ce paragraphe rappeler la construction due à Siegel (voir [10]) qui a été reprise par la suite par Shimura (voir [8]).

Soit $L_{1}$ une algèbre à division munie d'une involution positive $\rho_{1}$. Notons $K_{1}$ le centre de $L_{1}$ et $q_{1}^{2}=\left[L_{1}: K_{1}\right]$, puis

$$
F_{1}=\left\{x \in K_{1} ; \rho_{1}(x)=x\right\} \text {. }
$$

On sait d'après les travaux d'Albert (voir [8] pages 150-153) que $F_{1}$ est un corps de nombres totalement réel et on notera $g_{1}$ son degré. De plus on dispose de la classification suivante :

- Type I : $L_{1}=F_{1}$.

- Type II : $L_{1}$ est une algèbre de quaternions totalement indéfinie; i.e. une algèbre centrale simple $L_{1}$ sur $F_{1}$ telle que les composantes simples de $L_{1} \otimes \mathbb{Q} \mathbb{R}$ soient isomorphes à $M_{2}(\mathbb{R})$.

- Type III : $L_{1}$ est une algèbre de quaternions totalement définie; i.e. une algèbre centrale simple $L_{1}$ sur $F_{1}$ telle que les composantes simples de $L_{1} \otimes_{\mathbb{Q}} \mathbb{R}$ soient isomorphes à l'algèbre $\mathbb{H}$ des quaternions hamiltoniens.

- Type IV : $L_{1}$ est une algèbre centrale simple sur $K_{1}$ et $K_{1}$ est une extension quadratique imaginaire de $F_{1}$. 
Plus précisément on a :

$$
L_{1} \otimes_{\mathbb{Q}} \mathbb{R} \simeq \begin{array}{ll}
\mathbb{R}^{g_{1}} & \text { si } L_{1} \text { est de type I, } \\
M_{2}(\mathbb{R})^{g_{1}} & \text { si } L_{1} \text { est de type II, } \\
\mathbb{H}^{g_{1}} & \text { si } L_{1} \text { est de type III, } \\
M_{q_{1}}(\mathbb{C})^{g_{1}} & \text { si } L_{1} \text { est de type IV. }
\end{array}
$$

En plongeant $\mathbb{H}$ de manière canonique dans $M_{2}(\mathbb{C})$, on obtient alors $g_{1}$ (resp. $2 g_{1}$ ) représentations matricielles irréductibles de $L_{1}$ dans le cas de type I, II ou III (resp. dans le cas de type IV), que l'on notera $\chi_{1}, \ldots, \chi_{g_{1}}$ (resp. $\chi_{1}, \ldots, \chi_{g_{1}}, \bar{\chi}_{1}, \ldots, \bar{\chi}_{g_{1}}$ ).

Soit $n$ un entier naturel tel que $2 n$ soit un multiple de $\left[L_{1}: \mathbb{Q}\right]$. On pose :

$$
m_{1}=\frac{2 n}{\left[L_{1}: \mathbb{Q}\right]} \text {. }
$$

On se donne en plus, dans le cas où $L_{1}$ est de type IV, un couple d'entiers $\left(r_{\nu}^{1}, s_{\nu}^{1}\right)$ pour tout $\nu$ compris entre 1 et $g_{1}$, vérifiant

$$
r_{\nu}^{1}+s_{\nu}^{1}=m_{1} q_{1} \text {. }
$$

On pose alors

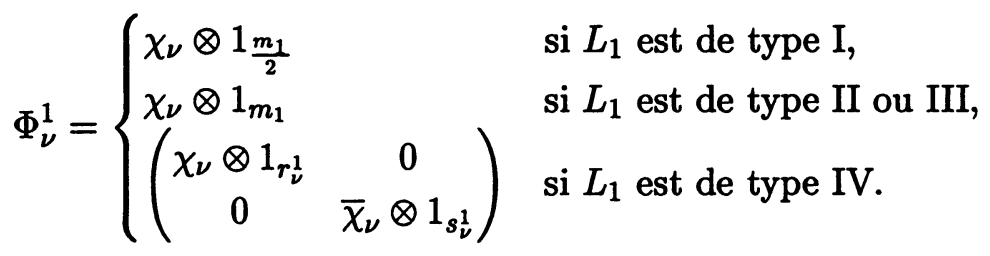

pour tout $\nu=1, \ldots, g_{1}$, puis

$$
\Phi^{1}=\left(\begin{array}{ccccc}
\Phi_{1}^{1} & 0 & \ldots & \ldots & 0 \\
0 & \ddots & \ddots & \ddots & \vdots \\
\vdots & \ddots & \Phi_{\nu} & \ddots & \vdots \\
\vdots & \ddots & \ddots & \ddots & 0 \\
0 & \ldots & \ldots & 0 & \Phi_{g}^{1}
\end{array}\right)
$$

On appellera $\Phi^{1}$ la représentation complexe canonique admissible associée à $L_{1}, n$, et aux $g_{1}$ couples $\left(r_{\nu}^{1}, s_{\nu}^{1}\right)$ si $L_{1}$ est de type IV.

Soit $A$ une variété abélienne de dimension $n$ définie sur $\mathbb{C}, L=\operatorname{End}^{0}(A)$. On sait que $A(\mathbb{C}) \simeq \mathbb{C}^{n} / D$ où $D$ est un réseau de $\mathbb{C}^{n}$, et qu'un élément de $L$ s'identifie à un endomorphisme $\mathbb{C}$-linéaire de $\mathbb{C}^{n}$ préservant $D \otimes_{\mathbb{Z}} \mathbb{Q}$. D'où un morphisme d'algèbres

$$
\Phi: L \rightarrow M_{n}(\mathbb{C})
$$

que l'on appelle représentation complexe analytique de $L$. 
Supposons maintenant que $A$ soit simple, $L$ est alors une algèbre à division et $D \otimes_{\mathbb{Z}} \mathbb{Q}$ est un $L$-espace vectoriel, en particulier $[L: \mathbb{Q}]$ divise $2 n$. On notera

$$
m=\frac{2 n}{[L: \mathbb{Q}]} .
$$

Soit $\mathcal{C}$ une polarisation de la variété abélienne $A$. On sait (voir [8] page 154) que la polarisation $\mathcal{C}$ induit une involution positive $\rho$ sur $L$. Par suite, nous sommes dans le cadre étudié au début de ce paragraphe, ce qui nous permet d'introduire les sous-corps $K$ et $F$, ainsi que les entiers $q$ et $g$. On vérifie en fait que $q$ et $g$ ne dépendent que de la variété abélienne $A$, et non du choix particulier de la polarisation $\mathcal{C}$.

Théorème 2.1. Soit $\Phi$ une représentation complexe analytique de $L=$ End ${ }^{0}(A)$, et soit $L_{1}$ une sous-algèbre de $L$, alors $\Phi_{/ L_{1}}$ est $\mathbb{C}$-équivalente à une et à une seule représentation complexe canonique admissible associée $\grave{a} L_{1}$ et $\grave{a} n$.

Référence. Voir [8] pages 155-156.

Corollaire 2.2. Lorsque $L$ est de type $I V$, la représentation complexe canonique admissible $\Phi$ de $L$ fournit $g$ couples d'entiers $\left(r_{\nu}, s_{\nu}\right)$ soumis à la condition $r_{\nu}+s_{\nu}=m q$. Ces $g$ couples d'entiers ne sont pas uniquement déterminés, par contre le cardinal de l'ensemble

$$
\left\{\nu \in\{1, \ldots, g\} ; r_{\nu} s_{\nu}=0\right\}
$$

ne dépend que de $A$, ce qui permet d'introduire la définition suivante.

Définition. A la variété abélienne simple $A$ de dimension $n$ on associe

$$
c= \begin{cases}0 & \text { si } L \text { est de type I-II ou III, } \\ \operatorname{Card}\left\{\nu \in\{1, \ldots, g\} ; r_{\nu} s_{\nu}=0\right\} & \text { si } L \text { est de type IV. }\end{cases}
$$

Remarque. En fait l'entier $c$ ne dépend que de la classe d'isogénie de la variété abélienne $A$. Cet entier $c$ mesure la "tendance à la multiplication complexe" de $A$ comme le montre le lemme suivant :

Lemme 2.3. Soit $A$ une variété abélienne simple de dimension $n$. Alors $A$ est à multiplication complexe si et seulement si $c=g$.

Référence. Voir le lemme 2.4 de [3].

Nous désignerons par $S\left(L_{1}, \Phi^{1}, \rho_{1}\right)$ l'ensemble des triplets $(A, \mathcal{C}, \theta)$, où $A$ est une variété abélienne de dimension $n$ définie sur $\mathbb{C}, \theta$ un morphisme d'algèbres injectif de $L_{1}$ dans $\operatorname{End}^{0}(A)$ vérifiant $\Phi \circ \theta \sim \mathbb{C} \Phi^{1}$ où $\Phi$ est une représentation complexe analytique de $\operatorname{End}^{0}(A)$, et $\mathcal{C}$ une polarisation de $A$ tel que la restriction de l'involution positive induite par $\mathcal{C}$ sur $\operatorname{End}^{0}(A)$ coïncide sur $\theta\left(L_{1}\right)$ avec l'involution $\theta(x) \mapsto \theta\left(x^{\rho_{1}}\right)$. 
A partir d'un élément $(A, \mathcal{C}, \theta)$ appartenant à $S\left(L_{1}, \Phi^{1}, \rho_{1}\right)$, Goro Shimura construit une matrice $T_{1} \in \mathrm{Gl}_{m_{1}}\left(L_{1}\right)$ vérifiant $T_{1}^{\rho_{1}}=-T_{1}$ et $\mathcal{M}_{1}$ un sous-Z-module libre de $L_{1}^{m_{1}}$ de rang $2 n$ (voir [8] page 157 pour les détails).

Le groupe algébrique réductif connexe défini sur $\mathbb{Q}$ associé est alors

$$
G\left(T_{1}\right)=\left\{U \in M_{m_{1}}\left(L_{1}\right) ; U T_{1} U^{\rho_{1}}=T_{1}\right\} .
$$

Lemme 2.4. On a $G\left(T_{1}\right)_{\mathbb{R}}$ est isomorphe à $S l_{2, \mathbb{R}} \times S l_{2, \mathbb{R}}$ (qui est le cas qui nous intéressera par la suite) si et seulement si on se trouve dans l'un des trois cas suivants :

- $n=2$ et $L_{1}$ corps réel de degré 2.

- $n=4$ et $L_{1}$ algèbre à division de type II et de degré 8 .

- $n=4, L_{1}$ corps C.M. de degré 4 et $r_{1}^{1}=s_{1}^{1}=1$.

Preuve du lemme 2.4. évident.

Notons $\Sigma\left(T_{1}, \mathcal{M}_{1}\right)$ une famille analytique d'éléments de $S\left(L_{1}, \Phi^{1}, \rho_{1}\right)$ comme dans [8]. Le résultat principal de Shimura dit que $\Sigma\left(T_{1}, \mathcal{M}_{1}\right)$ est paramétré par l'ensemble des points d'un domaine symétrique borné complexe $\mathfrak{H}\left(\Phi^{1}\right)$ où

avec

$$
\mathfrak{H}\left(\Phi^{1}\right)=\Pi_{1 \leq \nu \leq g_{1}} \mathfrak{H}\left(\Phi_{\nu}^{1}\right)
$$

$$
\mathfrak{H}\left(\Phi_{\nu}^{1}\right)= \begin{cases}\mathfrak{H}_{\frac{m_{1}}{1}}^{1} & \text { si } L_{1} \text { est de type I, } \\ \mathfrak{H}_{m_{1}}^{1} & \text { si } L_{1} \text { est de type II } \\ \mathfrak{H}_{m_{1}}^{2} & \text { si } L_{1} \text { est de type III } \\ \mathfrak{H}_{r_{\nu}^{1}, s_{\nu}^{1}}^{3} & \text { si } L_{1} \text { est de type IV }\end{cases}
$$

et

$$
\begin{aligned}
& \mathfrak{H}_{t}^{1}=\left\{z \in M_{t}(\mathbb{C}) ;{ }^{t} z=z, I_{t}-z^{t} \bar{z} \text { positive hermitienne }\right\} \\
& \mathfrak{H}_{t}^{2}=\left\{z \in M_{t}(\mathbb{C}) ;{ }^{t} z=-z, I_{t}-z^{t} \bar{z} \text { positive hermitienne }\right\} \\
& \mathfrak{H}_{r, s}^{3}=\left\{z \in M_{r, s}(\mathbb{C}) ; I_{r}-z^{t} \bar{z} \text { positive hermitienne }\right\}
\end{aligned}
$$

Remarques. 1) Dans le cas où $r s=0$, l'espace $\mathfrak{H}_{r, s}^{3}$ n'a aucune signification. On convient alors que $\mathfrak{H}_{r, s}^{3}$ est réduit à un point.

2) Bien que l'espace $\mathfrak{H}\left(\Phi^{1}\right)$ ne possède pas de $\overline{\mathbb{Q}}$-structure, on posera

$$
\mathfrak{H}_{t}^{i}(\overline{\mathbb{Q}})=\mathfrak{H}_{t}^{i} \cap M_{t}(\overline{\mathbb{Q}})
$$

pour tout $i=1,2$ et

$$
\mathfrak{H}_{r, s}^{3}(\overline{\mathbb{Q}})=\mathfrak{H}_{r, s}^{3} \cap M_{r, s}(\overline{\mathbb{Q}})
$$

ce qui permet de définir de manière naïve $\mathfrak{H}\left(\Phi^{1}\right)(\overline{\mathbb{Q}})$.

3) Lorsque $t=1, \mathfrak{H}_{t}^{1}$ n'est autre que $\mathfrak{B}=B O(O, 1)$ la boule ouverte de $\mathbb{C}$ de centre l'origine et de rayon 1 . On rappelle que $\mathfrak{B}$ est biholomorphiquement isomorphe à $\mathfrak{H}$ le demi-plan supérieur de Poincaré (via $z \rightarrow \frac{z-i}{z+i}$ ). 
Notez que cette transformation envoie les points algébriques sur les points algébriques.

4) On a $G\left(T_{1}\right)_{\mathbb{R}} \simeq \mathrm{Sl}_{2, \mathbb{R}} \times \mathrm{Sl}_{2, \mathbb{R}}$ si et seulement si $\mathfrak{H}\left(\Phi^{1}\right)=\mathfrak{B} \times \mathfrak{B}$.

De plus, il existe un groupe arithmétique $\Gamma\left(T_{1}, \mathcal{M}_{1}\right)$ de $G\left(T_{1}\right)$ agissant discontinument sur $\mathfrak{H}\left(\Phi^{1}\right)$, tel que l'ensemble quotient $\Gamma\left(T_{1}, \mathcal{M}_{1}\right) \backslash \mathfrak{H}\left(\Phi^{1}\right)$ soit en bijection avec l'ensemble des classes d'isomorphisme d'éléments de $\Sigma\left(T_{1}, \mathcal{M}_{1}\right)$.

Nous prions le lecteur de se référer à l'article de [8] pour les détails. Indiquons brièvement la construction de la variété abélienne sous-jacente à un triplet appartenant à $\Sigma\left(T_{1}, \mathcal{M}_{1}\right)$ et associé à un point $z \in \mathfrak{H}\left(\Phi^{1}\right)$.

On commence par construire $r_{1}^{\nu}\left(z_{\nu}, T_{1}\right), \ldots, r_{m_{1}}^{\nu}\left(z_{\nu}, T_{1}\right)$ des éléments de $\mathbb{C}^{\frac{n}{g_{1}}}$, puis on pose

$$
r_{k}\left(z, T_{1}\right)={ }^{t}\left(r_{k}^{1}\left(z_{1}, T_{1}\right), \ldots, r_{k}^{g_{1}}\left(z_{g_{1}}, T_{1}\right)\right) .
$$

On vérifie alors que les vecteurs $\left(r_{1}, \ldots, r_{m_{1}}\right)$ sont $\Phi^{1}\left(L_{1}\right)$-linéairement indépendants. On pose alors

$$
D\left(z, T_{1}, \mathcal{M}_{1}\right)=\left\{\sum_{i=1}^{m_{1}} \Phi^{1}\left(a_{i}\right) r_{i}\left(z, T_{1}\right) ;\left(a_{1}, \ldots, a_{m_{1}}\right) \in \mathcal{M}_{1}\right\} .
$$

Si $A$ désigne la variété abélienne sous-jacente à l'élément de $\Sigma\left(T_{1}, \mathcal{M}_{1}\right)$ associée à $z \in \mathfrak{H}\left(\Phi^{1}\right)$, alors

$$
A(\mathbb{C}) \simeq \mathbb{C}^{n} / D\left(z, T_{1}, \mathcal{M}_{1}\right) .
$$

Notations. Si $L_{1}$ est de type I, II, III on notera parfois $D_{n}\left(L_{1}\right)$ au lieu de $\mathfrak{H}\left(\Phi^{1}\right)$, et $S_{n}\left(L_{1}, \rho_{1}\right)$ au lieu de $S\left(L_{1}, \Phi^{1}, \rho_{1}\right)$. Bien entendu $\Phi^{1}$ désigne la représentation canonique admissible asșociée à $L_{1}$ et à $n$. Si on ne désire pas préciser une famille analytique $\Sigma\left(T_{1}, \mathcal{M}_{1}\right)$ incluse dans $S\left(L_{1}, \Phi^{1}, \rho_{1}\right)$, on la notera plutôt $\Sigma_{n}\left(L_{1}, \rho_{1}\right)$.

Si $L_{1}$ est de type IV, étant donné pour tout $\nu$ compris entre 1 et $g_{1}$ un couple $\left(r_{\nu}^{1}, s_{\nu}^{1}\right)$ vérifiant $r_{\nu}^{1}+s_{\nu}^{1}=m_{1} q_{1}$, on notera parfois $D_{n}\left(L_{1}, r_{1}^{1}, \ldots, r_{g_{1}}^{1}\right)$ au lieu de $\mathfrak{H}\left(\Phi^{1}\right)$, et $S_{n}\left(L_{1}, r_{1}^{1}, \ldots, r_{g_{1}}^{1}, \rho_{1}\right)$ au lieu de $S\left(L_{1}, \Phi^{1}, \rho_{1}\right)$. Ici encore $\Phi^{1}$ désigne la représentation complexe canonique admissible associée à $L_{1}$, $n$ et aux couples $\left(r_{\nu}^{1}, s_{\nu}^{1}\right)$ pour tout $1 \leq \nu \leq g_{1}$. De même, si on ne désire pas préciser une famille analytique $\Sigma\left(T_{1}, \mathcal{M}_{1}\right)$ incluse dans $S\left(L_{1}, \Phi^{1}, \rho_{1}\right)$, on la notera plutôt $\Sigma_{n}\left(L_{1}, r_{1}^{1}, \ldots, r_{g_{1}}^{1}, \rho_{1}\right)$.

De plus, lorsqu'il n'existe qu'une seule involution positive $\rho_{1}$ sur $L_{1}$, nous ne ferons pas toujours figurer $\rho_{1}$ dans les notations.

Le résultat suivant sera souvent utilisé par la suite : 
Lemme 2.5. Soient $(A, \mathcal{C}, \theta) \in S\left(L_{1}, \Phi^{1}, \rho_{1}\right)$ et $\left(A^{\prime}, \mathcal{C}^{\prime}, \theta^{\prime}\right) \in S\left(L_{1}, \Phi^{1}, \rho_{1}^{\prime}\right)$ et supposons que les variétés abéliennes $A$ et $A^{\prime}$ soient isogènes. Désignons par $z \in \mathfrak{H}\left(\Phi^{1}\right)$ (resp. par $z^{\prime} \in \mathfrak{H}\left(\Phi^{1}\right)$ ) un représentant de l'élément de

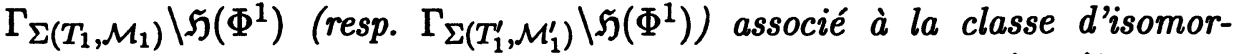
phisme de $(A, \mathcal{C}, \theta) \in \Sigma\left(T_{1}, \mathcal{M}_{1}\right)$ (resp. $\left(A^{\prime}, \mathcal{C}^{\prime}, \theta^{\prime}\right) \in \Sigma\left(T_{1}^{\prime}, \mathcal{M}_{1}^{\prime}\right)$. Alors $z_{\nu} \in \mathfrak{H}\left(\Phi_{\nu}^{1}\right)(\overline{\mathbb{Q}})$ si et seulement si $z_{\nu}^{\prime} \in \mathfrak{H}\left(\Phi_{\nu}^{1}\right)(\overline{\mathbb{Q}})$.

Référence. Bien que ce résultat ne figure pas explicitement dans l'article de Shimura, il est facile de le déduire à partir des considérations faites page 161-163 de [8].

Le lemme précédent nous permet de poser la définition suivante :

Définition. Étant donné $[A]$ une $\mathbb{C}$-classe d'isogénie de variétés abéliennes telle que $L_{1}$ s'injecte dans $\operatorname{End}^{0}(A)$, on associe $e\left(L_{1},[A]\right) \in\left(\mathbb{F}_{2}\right)^{g_{1}}$ défini de la manière suivante : étant donnée $\mathcal{C}$ une polarisation de $A$ et $\theta$ un morphisme d'algèbres injectif de $L_{1}$ dans $\operatorname{End}^{0}(A)$, le triplet $(A, \mathcal{C}, \theta)$ appartient à une famille analytique $\Sigma\left(T_{1}, \mathcal{M}_{1}\right)$ incluse dans $S\left(L_{1}, \Phi^{1}, \rho_{1}\right)$ et paramétrée par $\mathfrak{H}\left(\Phi^{1}\right)$. Si $z \in \mathfrak{H}\left(\Phi^{1}\right)$ désigne un représentant de la classe de $\Gamma\left(T_{1}, \mathcal{M}_{1}\right) \backslash \mathfrak{H}\left(\Phi^{1}\right)$ associé à la classe d'isomorphisme de l'élément $(A, \mathcal{C}, \theta) \in$ $\Sigma\left(T_{1}, \mathcal{M}_{1}\right)$, on pose :

$$
e\left(L_{1},[A]\right)_{\nu}= \begin{cases}0 & \text { si } z_{\nu} \in \mathfrak{H}\left(\Phi_{\nu}^{1}\right)(\overline{\mathbb{Q}}) \\ 1 & \text { sinon }\end{cases}
$$

pour tout $\nu=1, \ldots, g_{1}$.

\section{3. Étude de la simplicité de certaines variétés abéliennes.}

Commençons par la proposition suivante qui est la clef de voûte de ce paragraphe.

Proposition 3.1. Soit $\left(A_{1}, \mathcal{C}_{1}, \theta_{1}\right)$ un triplet appartenant à une famille analytique $\Sigma_{1}$ incluse dans $S\left(L_{1}, \Phi^{1}, \rho_{1}\right)$ telle que $\mathfrak{H}\left(\Phi^{1}\right)=\mathfrak{B} \times \mathfrak{B}$. Supposons que $A_{1}$ soit isogène à une puissance pure d'une variété abélienne simple $A_{2}$ i.e. $A_{1}$ isogène à $A_{2}^{u}$. Alors, si $\mathfrak{H}\left(\Phi^{2}\right)=\mathfrak{B}$, où $\Phi^{2}$ est la représentation complexe canonique admissible associée à $A_{2}$, on a

$$
e\left(L_{1},\left[A_{1}\right]\right)_{1}=e\left(L_{1},\left[A_{1}\right]\right)_{2} .
$$

Preuve de la proposition 3.1. Soit $\mathcal{C}_{2}$ la polarisation de $A_{2}$ induite par $\mathcal{C}_{1}$ et l'isogénie. Posons $L_{2}=\operatorname{End}^{0}\left(A_{2}\right), \theta_{2}=I d_{L_{2}}$ et $i: L_{1} \hookrightarrow M_{u}\left(L_{2}\right)$ l'injection déduite de $\theta_{1}: L_{1} \hookrightarrow \operatorname{End}^{0}\left(A_{1}\right)$ et de l'isomorphisme naturel $\operatorname{End}^{0}\left(A_{1}\right) \simeq M_{u}\left(\operatorname{End}^{0}\left(A_{2}\right)\right)$.

Le triplet $\left(A_{2}, \mathcal{C}_{2}, \theta_{2}\right)$ appartient à une famille analytique disons $\Sigma_{2}$ incluse dans $S\left(L_{2}, \Phi^{2}, \rho_{2}\right)$. Notons $\tau \in \mathfrak{B}$ un représentant de $\left(A_{2}, \mathcal{C}_{2}, \theta_{2}\right) \in \Sigma_{2}$. 
Soit $\left(A_{1}^{\prime}, \mathcal{C}_{1}^{\prime}, \theta_{1}^{\prime}\right)$ l'image de $\left(A_{2}, \mathcal{C}_{2}, \theta_{2}\right)$ par l'injection canonique $\Sigma_{2} \hookrightarrow \Sigma_{1}^{\prime}$ i.e. $A_{1}^{\prime}=A_{2}^{u}, \theta_{1}=M_{u}\left(\theta_{2}\right) \circ i$ et $\mathcal{C}_{1}^{\prime}$ la polarisation de $A_{1}^{\prime}$ induite par $\mathcal{C}_{2}$. On sait (voir [6] page 85) qu'il existe $a^{\prime}$ et $a^{\prime \prime}$ deux nombres complexes ne dépendant que de $\Sigma_{1}^{\prime}$ et $\Sigma_{2}$ tel que $\left(A_{1}^{\prime}, \mathcal{C}_{1}^{\prime}, \theta_{1}^{\prime}\right) \in \Sigma_{1}^{\prime}$ soit représenté par $\left(z_{1}^{\prime}, z_{1}^{\prime \prime}\right) \in \mathfrak{B} \times \mathfrak{B}$ où

$$
\left\{\begin{array}{l}
z_{1}^{\prime}=a^{\prime} \tau, \\
z_{1}^{\prime \prime}=a^{\prime \prime} \tau .
\end{array}\right.
$$

Je prétends maintenant que $a^{\prime}$ et $a^{\prime \prime}$ sont des nombres algébriques. En effet, si $A_{2}$ est à multiplication complexe alors $A_{1}^{\prime}$ est aussi à multiplication complexe et les nombres $\tau, z^{\prime}, z^{\prime \prime}$ sont algébriques non nuls.

Par suite $z_{1}^{\prime} \in \overline{\mathbb{Q}}$ si et seulement si $z_{1}^{\prime \prime} \in \overline{\mathbb{Q}}$. Comme $A_{1}^{\prime} \in\left[A_{1}\right]$, on a bien $e\left(L_{1},\left[A_{1}\right]\right)_{1}=e\left(L_{1},\left[A_{1}\right]\right)_{2}$ d'après le lemme 2.5.

Jusqu'à la fin de l'article, nous identifierons $\mathfrak{B}$ avec $\mathfrak{H}$, via le biholomorphisme décrit précédemment. Nous allons maintenant donner quelques applications de la proposition 3.1 .

Lemme 3.2. Soit $L_{1}$ un corps totalement réel de degré 2 . Notons $\Sigma_{2}\left(L_{1}\right)$ une famille analytique incluse dans $S_{2}\left(L_{1}\right)$ paramétrée par $D_{2}\left(L_{1}\right)=\mathfrak{H} \times \mathfrak{H}$. Soit $z=\left(z_{1}, z_{2}\right) \in \mathfrak{H} \times \mathfrak{H}$ tel que l'un des deux nombres complexes $z_{1}$ ou $z_{2}$ soit algébrique l'autre étant transcendant, alors la surface abélienne $A$ sous-jacente au triplet associé à $z=\left(z_{1}, z_{2}\right)$ et appartenant à $\Sigma_{2}\left(L_{1}\right)$ est simple.

Preuve du lemme 3.2. Raisonnons par l'absurde et supposons que $A$ soit non simple. $\mathrm{D}$ 'après le théorème de réductibilité de Poincaré, la variété abélienne $A$ est isogène à $A_{1}^{2}$ où $A_{1}$ est une courbe elliptique. Comme $D_{1}(\mathbb{Q})=\mathfrak{H}$, la situation est redevable de la proposition 3.1 et on déduit que les deux nombres complexes $z_{1}$ et $z_{2}$ sont soit simultanément algébriques, soit simultanément transcendants. Contradiction.

Lemme 3.3. Soient $L_{1}$ une algèbre à division de type II et de degré 8 , et $\rho_{1}$ une involution positive sur $L_{1}$. Notons $\Sigma_{4}\left(L_{1}, \rho_{1}\right)$ une famille analytique incluse dans $S_{4}\left(L_{1}, \rho_{1}\right)$ paramétrée par $D_{4}\left(L_{1}\right)=\mathfrak{H} \times \mathfrak{H}$. Soit $z=\left(z_{1}, z_{2}\right) \in$ $\mathfrak{H} \times \mathfrak{H}$ tel que l'un des deux nombres complexes $z_{1}$ ou $z_{2}$ est algébrique, l'autre étant transcendant, alors la variété abélienne sous-jacente au triplet associé à $z=\left(z_{1}, z_{2}\right)$ et appartenant à $\Sigma_{4}\left(L_{1}, \rho_{1}\right)$ est simple.

Preuve du lemme 3.3. Raisonnons par l'absurde et supposons que $A$ est non simple. Par le théorème de réductibilité de Poincaré $A$ est nécessairement isogène à $A_{1}^{\alpha_{1}}$, où $A_{1}$ est une variété abélienne simple de dimension 1 ou 2.

On montre que le cas $\operatorname{dim} A_{1}=1$ est à exclure à l'aide d'un argument similaire à celui donné au théorème précédent. Ainsi nécessairement $\operatorname{dim} A_{1}=2$, et $A$ est isogène à $A_{1}^{2}$. 
Connaissant les différentes possibilités pour l'algèbre des endomorphismes d'une surface abélienne et tenant compte du fait que $L_{1}$ s'injecte dans $M_{2}\left(\operatorname{End}^{0}\left(A_{1}\right)\right)$, on déduit que nécessairement $\operatorname{End}^{0}\left(A_{1}\right)$ ne peut être qu'un corps C.M. de degré 4 ou une algèbre de type II et de degré 4 . En fait, il est clair que $\operatorname{End}^{0}\left(A_{1}\right)$ ne peut être un corps C.M. de degré 4 , en effet $A_{1}$ serait à multiplication complexe et il en serait donc de même pour la variété abélienne $A$ ce qui implique en particulier que les nombres complexes $z_{1}$ et $z_{2}$ sont tous les deux algébriques. Absurde.

Ainsi $L_{1}^{\prime}=\operatorname{End}^{0}\left(A_{1}\right)$ est une algèbre à division de type II et de degré 4 . Comme $D_{2}\left(L_{1}^{\prime}\right)=\mathfrak{H}$, on déduit d'après la proposition 3.1 que les nombres complexes $z_{1}$ et $z_{2}$ sont soit simultanément algébriques, soit simultanément transcendants. Contradiction.

Lemme 3.4. Soit $L_{1}$ un corps C.M. de degré 4. Notons $\Sigma_{4}\left(L_{1}, 1,1\right)$ une famille analytique incluse dans $S_{4}\left(L_{1}, 1,1\right)$ paramétrée par $D_{4}\left(L_{1}, 1,1\right)=$ $\mathfrak{H} \times \mathfrak{H}$. Soit $z=\left(z_{1}, z_{2}\right) \in \mathfrak{H} \times \mathfrak{H}$ tel que l'un des deux nombres complexes $z_{1}$ ou $z_{2}$ est algébrique, l'autre transcendant. Alors la variété abélienne $A$ sous-jacente au triplet associé à $z=\left(z_{1}, z_{2}\right)$ et appartenant $\grave{a} \Sigma_{4}\left(L_{1}, 1,1\right)$ est simple. De plus $L=E n d^{0}(A)$ ne peut pas être une algèbre à division non commutative de type $I V$ et de degré 8.

Preuve du lemme 3.4. Commençons par montrer que $A$ est simple. Pour cela raisonnons par l'absurde et supposons que cela ne soit pas le cas. De la même manière que précédemment, on commence par vérifier que $A$ est isogène à une puissance pure d'une variété abélienne simple $A_{1}$. En effet, sinon $A$ serait isogène à un produit de deux surfaces abéliennes simples à multiplication complexe, ce qui impliquerait que $A$ est à multiplication complexe et les nombres complexes $z_{1}$ et $z_{2}$ seraient algébriques.

Il est clair que le cas $\operatorname{dim} A_{1}=1$ est à exclure, en effet $D_{1}(\mathbb{Q})=\mathfrak{H}$ et la situation est redevable de la proposition 3.1.

Ainsi nécessairement $\operatorname{dim} A_{1}=2$ et $\operatorname{End}^{0}\left(A_{1}\right)$ ne peut être qu'un corps totalement réel de degré 2 , un corps C.M. de degré 2 ou 4 , une algèbre à division de type II et de degré 4 . En appliquant une nouvelle fois la proposition 3.1, on voit qu'il est impossible que $\operatorname{End}^{0}\left(A_{1}\right)$ soit un corps C.M. de degré 2 ou même une algèbre à division de type II et de degré 4 . Par ailleurs, si $\operatorname{End}^{0}\left(A_{1}\right)$ est un corps C.M. de degré 4, alors $A_{1}$ est à multiplication complexe, et il en est donc de même pour $A$. D'où l'algébricité de $z_{1}$ et de $z_{2}$, ce qui contredit les hypothèses. Ainsi $\operatorname{End}^{0}\left(A_{1}\right)$ ne peut être qu'un corps totalement réel $F_{1}$ de degré 2 , et l'élément $(A, \mathcal{C}, \theta) \in \Sigma_{4}\left(L_{1}, 1,1\right)$ est isogène au carré d'un élément appartenant à $\Sigma_{2}\left(F_{1}\right)$. Vu le lemme 2.5 , on peut même supposer que l'élément $(A, \mathcal{C}, \theta) \in \Sigma_{4}\left(L_{1}, 1,1\right)$ est isomorphe au carré d'un élément appartenant à $\Sigma_{2}\left(F_{1}\right)$.

De plus, on sait d'après [6] page 85 qu'il existe $\left(a_{1}, b_{1}, a_{2}, b_{2}\right)$ un quadruplet de nombres algébriques tel que si un élément de $\Sigma_{2}\left(F_{1}\right)$ est représenté 
$\operatorname{par} \tau=\left(\tau_{1}, \tau_{2}\right) \in \mathfrak{H} \times \mathfrak{H}$, alors le carré de cet élément, vu comme un élément de $\Sigma_{4}\left(L_{1}, 1,1\right)$, est représenté par $z=\left(z_{1}(\tau), z_{2}(\tau)\right) \in \mathfrak{H} \times \mathfrak{H}$ où

Notons

$$
\left\{\begin{array}{l}
z_{1}(\tau)=a_{1} \tau_{1}+a_{2} \tau_{2} \\
z_{2}(\tau)=b_{1} \tau_{1}+b_{2} \tau_{2}
\end{array}\right.
$$

$$
M=\left(\begin{array}{ll}
a_{1} & a_{2} \\
b_{1} & b_{2}
\end{array}\right) \in M_{2}(\overline{\mathbb{Q}}) .
$$

La matrice $M$ ne peut pas être inversible. Sinon, il existerait $\tau \in \mathfrak{H} \times \mathfrak{H}$ tel que $\left(z_{1}(\tau), z_{2}(\tau)\right) \in \mathfrak{H} \times \mathfrak{H}$ soit un point générique pour les fonctions $f_{1}, \ldots, f_{k}$ définies au théorème 3 de l'article de [8] page 169 relativement à la famille analytique $\Sigma_{4}\left(L_{1}, 1,1\right)$. Par le préambule du $\S 4$ page 176 de l'article de [8], l'élément $(A, \mathcal{C}, \theta) \in \Sigma_{4}\left(L_{1}, 1,1\right)$ associé à $\left(z_{1}(\tau), z_{2}(\tau)\right)$ est générique. Or d'après le théorème 5 page 176 de l'article de [8], on déduit que $\operatorname{End}^{0}(A) \simeq L_{1}$. Ce qui prouve en particulier que la variété abélienne $A$ est simple. Contradiction. Ainsi la matrice $M \in M_{2}(\overline{\mathbb{Q}})$ est singulière, et par suite

$$
b_{1} z_{1}=a_{1} z_{2}
$$

et

$$
b_{2} z_{1}=a_{2} z_{2} \text {. }
$$

Les quatre nombres algébriques $a_{1}, a_{2}, b_{1}$ et $b_{2}$ ne peuvent être tous nuls. D'où les nombres complexes $z_{1}$ et $z_{2}$ sont soit simultanément algébriques, soit simultanément transcendants. Nous obtenons donc une nouvelle contradiction. Ainsi, la variété abélienne $A$ est simple.

Supposons maintenant que $L=\operatorname{End}^{0}(A)$ soit une algèbre à division non commutative de type IV et de degré 8 . Alors comme $D_{4}\left(L_{1}, 1\right)=\mathfrak{H}$ on déduit, d'après la proposition 3.1 que les nombres complexes $z_{1}$ et $z_{2}$ sont soit simultanément algébriques, soit simultanément transcendants. Ce qui est impossible.

\section{Contrôle de l'algébricité de $z$}

Soit $n$ un entier strictement positif, $A$ une variété abélienne de dimension $n$ définie sur $\mathbb{C}$. Notons $\Omega \in M_{n, 2 n}(\mathbb{C})$ une matrice des périodes rationnelles de $A$ (i.e. obtenue à partir d'une base du $\mathbb{Q}$-espace vectoriel $D \otimes_{\mathbb{Z}} \mathbb{Q}$ ). On notera dans tout ce qui suit :

$$
V=M_{2 n, 1}(\overline{\mathbb{Q}})=\overline{\mathbb{Q}}^{2 n}
$$

et

Soit

$$
V_{\mathbb{C}}=V \otimes_{\overline{\mathbb{Q}}} \mathbb{C}=M_{2 n, 1}(\mathbb{C})=\mathbb{C}^{2 n}
$$

$$
V_{\Omega}=\left\{E \in V_{\mathbb{C}} ; \Omega E=0_{M_{n, 1}(\mathbb{C})}\right\} .
$$


Alors $V_{\Omega}$ est un $\mathbb{C}$-sous-espace vectoriel de $V_{\mathbb{C}}$ de dimension $n$.

Nous avons démontré dans [3] le résultat suivant :

Théorème 4.1. Si la variété abélienne simple $A$ de dimension $n$ est définie sur $\overline{\mathbb{Q}}$, alors la codimension du plus petit $\mathbb{C}$-sous-espace vectoriel de $V_{\mathbb{C}}$ contenant $V_{\Omega}$ et défini sur $\overline{\mathbb{Q}}$ est égale $\grave{a} \frac{c}{g} n$.

Référence. Voir le théorème principal 2.2 figurant dans [3] à la page 22.

Le corollaire qui va suivre se trouve dans [4]. Par commodité pour le lecteur, nous reproduisons une preuve. Il convient d'autre part de rappeler que tout point d'un domaine symétrique complexe de dimension nulle est un point algébrique de ce domaine.

Corollaire 4.2. Les notations étant celles du préambule, soit $(A, \mathcal{C}, \theta)$ un élément de $\Sigma\left(T_{1}, \mathcal{M}_{1}\right)$ tel que $A$ soit une variété abélienne simple définie sur $\overline{\mathbb{Q}}$. Alors, si $z=\left(z_{1}, \ldots, z_{g_{1}}\right) \in \mathfrak{H}\left(\Phi^{1}\right)$ est un représentant de la classe $[z] \in \Gamma\left(T_{1}, \mathcal{M}_{1}\right) \backslash \mathfrak{H}\left(\Phi^{1}\right)$ associée à $(A, \mathcal{C}, \theta) \in \Sigma\left(T_{1}, \mathcal{M}_{1}\right)$, on $a$ :

$$
\text { Card }\left\{\nu \in\left\{1, \ldots, g_{1}\right\} ; z_{\nu} \in \mathfrak{H}\left(\Phi_{\nu}^{1}\right)(\overline{\mathbb{Q}})\right\} \leq E\left(\frac{c}{g} g_{1}\right),
$$

où $E(x)$ désigne la partie entière du nombre $x$.

En particulier, si $\theta$ réalise un isomorphisme entre $L_{1}$ et $E n d^{0}(A)$, alors pour tout indice $\nu$ compris entre 1 et $g$ tel que $\operatorname{dim}\left(\mathfrak{H}\left(\Phi_{\nu}^{1}\right)\right)>0$ on a $z_{\nu} \notin \mathfrak{H}\left(\Phi_{\nu}^{1}(\overline{\mathbb{Q}})\right)$.

Preuve du corollaire 4.2. Soit $\left(l_{1}, \ldots, l_{s}\right)$ une base de $L_{1}$ sur $\mathbb{Q}$. Posons $\lambda_{i+s(j-1)}=\Phi^{1}\left(l_{i}\right) r_{j}\left(z, T_{1}\right)$ pour tout $1 \leq i \leq s$ et $1 \leq j \leq m_{1}$.

Notons $\Omega={ }^{t}\left(\Omega_{1}, \ldots, \Omega_{g_{1}}\right)=\left(\lambda_{i k}\right)_{1 \leq i \leq n, 1 \leq k \leq 2 n} \in M_{n, 2 n}(\mathbb{C})$ la matrice des périodes ainsi obtenue et $I=\left\{\nu \in\left\{1, \ldots, g_{1}\right\} ; z_{\nu} \in \mathfrak{H}\left(\Phi_{\nu}^{1}\right)(\overline{\mathbb{Q}})\right\}$. On a $V_{\Omega} \subset W_{1}$ où

$$
W_{1}=\left\{E \in M_{2 n, 1}(\mathbb{C}) ; \Omega_{\nu} E=0_{M_{\frac{n}{g_{1}}, 1}(\mathbb{C})} \text { pour tout } \nu \in I\right\}
$$

De plus le $\mathbb{C}$-sous-espace vectoriel $W_{1}$ de $M_{2 n, 1}(\mathbb{C})$ est défini sur $\overline{\mathbb{Q}}$ (puisque $\Omega_{\nu} \in M_{\frac{n}{g_{1}}, 2 n}(\overline{\mathbb{Q}})$ pour tout $\left.\nu \in I\right)$ et est de codimension $\frac{n}{g_{1}} \operatorname{Card}(I)$. D'après le théorème 4.1 on déduit que $\frac{n}{g_{1}} \operatorname{Card}(I) \leq \frac{c}{g} n$, i.e. $\operatorname{Card}(I) \leq \frac{c}{g} g_{1}$.

\section{Applications}

Théorème 5.1. Soient $L_{1}$ une algèbre à division de type II et de degré 8 , et $\rho_{1}$ une involution positive sur $L_{1}$. Désignons par $\Sigma_{4}\left(L_{1}, \rho_{1}\right)$ une famille analytique d'éléments de $S_{4}\left(L_{1}, \rho_{1}\right)$ paramétrée par $\mathfrak{H} \times \mathfrak{H}$. Soit $z=$ $\left(z_{1}, z_{2}\right) \in \mathfrak{H} \times \mathfrak{H}$ tel que l'un des deux nombres complexes $z_{1}$ ou $z_{2}$ soit 
algébrique, l'autre transcendant. Alors le triplet $(A, \mathcal{C}, \theta)$ associé $a ̀ ~ z$ et appartenant à $\Sigma_{4}\left(L_{1}, \rho_{1}\right)$ n'est pas défini sur $\overline{\mathbb{Q}}$, ou ce qui revient au même

$$
J_{\Sigma_{4}\left(L_{1}, \rho_{1}\right)}(z) \notin V_{\Sigma_{4}\left(L_{1}, \rho_{1}\right)}(\overline{\mathbb{Q}}) \text {. }
$$

Avant de démontrer ce théorème nous avons besoin du résultat suivant :

Lemme 5.2. Soit $A$ une variété abélienne simple de dimension $n$ définie sur $\mathbb{C}$. $S$ 'il existe une sous-algèbre $L_{1}$ de $L=E n d^{0}(A)$ de degré $2 n$ alors $L_{1}=L$.

Preuve du lemme 5.2. Ce résultat résulte du fait que d'une part $D_{\mathbb{Q}}$ est un $L$-espace vectoriel, et que d'autre part $\operatorname{dim}_{\mathbb{Q}} D_{\mathbb{Q}}=2 n$.

Preuve du théorème 5.1. On sait, d'une part que $A$ est simple d'après les résultats du paragraphe 3 et d'autre part que $\theta\left(L_{1}\right)=\operatorname{End}^{0}(A)$ par le lemme 5.2.

Il est clair que le triplet $(A, \mathcal{C}, \theta) \in \Sigma_{4}\left(L_{1}, \rho_{1}\right)$ ne peut être défini sur $\overline{\mathbb{Q}}$. En effet, si tel était le cas la variété abélienne $A$ serait définie sur $\overline{\mathbb{Q}}$, et le corollaire 4.2 fournirait la transcendance des nombres complexes $z_{1}$ et $z_{2}$. D'où le résultat annoncé.

Théorème 5.3. Soit $L_{1}$ un corps de nombres totalement réel de degré 2. Désignons par $\Sigma_{2}\left(L_{1}\right)$ une famille analytique d'éléments de $S_{2}\left(L_{1}\right)$ paramétrée par $\mathfrak{H} \times \mathfrak{H}$. Etant donné $z=\left(z_{1}, z_{2}\right) \in \mathfrak{H} \times \mathfrak{H}$ tel que l'un des deux nombres $z_{1}$ ou $z_{2}$ soit algébrique et l'autre transcendant, le triplet $(A, C, \theta)$ associé à $z$ et appartenant à $\Sigma_{2}\left(L_{1}\right)$ n'est pas défini sur $\overline{\mathbb{Q}}$, ou ce qui revient au même

$$
J_{\Sigma_{2}\left(L_{1}\right)}(z) \notin V_{\Sigma_{2}\left(L_{1}\right)}(\overline{\mathbb{Q}}) .
$$

Preuve du théorème 5.3. D'après les résultats du paragraphe 3 , on sait que la variété abélienne $A$ est simple. Raisonnons par l'absurde et supposons que le triplet $(A, \mathcal{C}, \theta)$ est défini sur $\overline{\mathbb{Q}}$. Par le corollaire 4.2 , on sait que $\theta\left(L_{1}\right)$ s'injecte strictement dans $\operatorname{End}^{0}(A)$. D'où pour des raisons de dimension le degré de $\operatorname{End}^{0}(A)$ est égal à 4 . En appliquant une nouvelle fois le corollaire 4.2 on déduit que nécessairement $c>0$ et donc que $\operatorname{End}^{0}(A)$ est une algèbre de type IV. Ainsi $\left[\operatorname{End}^{0}(A): Z\left(\operatorname{End}^{0}(A)\right)\right]=1$ ou 2. Mais comme la dimension d'un corps gauche sur son centre est un carré parfait, on déduit que $\left[\operatorname{End}^{0}(A): Z\left(\operatorname{End}^{0}(A)\right)\right]=1$, i.e. $\operatorname{End}^{0}(A)=Z\left(\operatorname{End}^{0}(A)\right)$. Par suite $A$ est une variété abélienne de type C.M., $z$ est un point spécial du domaine $\mathfrak{H} \times \mathfrak{H}$ et les nombres complexes $z_{1}$ et $z_{2}$ sont tous les deux algébriques, ce qui fournit une contradiction et prouve le résultat annoncé.

Théorème 5.4. Soient $L_{1}$ un corps C.M. de degré 4 et $\Sigma_{4}\left(L_{1}, 1,1\right)$ une famille analytique de triplets incluse dans $S_{4}\left(L_{1}, 1,1\right)$. Etant donné $z=$ $\left(z_{1}, z_{2}\right) \in \mathfrak{H} \times \mathfrak{H}$ tel que l'un des deux nombres complexes $z_{1}$ ou $z_{2}$ est 
algébrique et l'autre transcendant, le triplet $(A, \mathcal{C}, \theta)$ associé à $z$ et appartenant à $\Sigma_{4}\left(L_{1}, 1,1\right)$ n'est pas défini sur $\overline{\mathbb{Q}}$, ou ce qui revient au même

$$
J_{\Sigma_{4}\left(L_{1}, 1,1\right)}(z) \notin V_{\Sigma_{4}\left(L_{1}, 1,1\right)}(\overline{\mathbb{Q}}) \text {. }
$$

Preuve du théorème 5.4. D'après le paragraphe 3 , on sait que la variété abélienne $A$ est simple. Raisonnons par l'absurde et supposons que le triplet $(A, \mathcal{C}, \theta)$ soit défini sur $\overline{\mathbb{Q}}$. Par une double application du corollaire 4.2, on sait que $\operatorname{End}^{0}(A)$ est une algèbre à division de type IV et de degré 8 sur $\mathbb{Q}$. L'algèbre $\operatorname{End}^{0}(A)$ est alors nécessairement commutative d'après le lemme 3.4. Par suite la variété abélienne $A$ est à multiplication complexe et les nombres complexes $z_{1}$ et $z_{2}$ seraient tous les deux algébriques. Ceci fournit une contradiction et prouve le résultat annoncé.

\section{Bibliographie}

[1] P.B. CoHEN, Humbert surfaces and transcendence properties of automorphic functions. Rocky Mountain J. of Math. 26 no. 3 (1996), 987-1001.

[2] P.B. Cohen, Propriétés transcendantes des fonctions automorphes. Séminaire de Théorie des Nombres, Paris, Cambridge University Press, 1992-93.

[3] G. Derome, Transcendance des valeurs des fonctions automorphes de Siegel. Journal of Number Theory 85 (2000), 18-34.

[4] G. Derome, Transcendance des valeurs des fonctions automorphes arithmétiques. Thèse de doctorat, Université des Sciences et Techniques de Lille.

[5] G. Faltings, Arithmetic varieties and rigidity. Séminaire de théorie des nombres de Paris, 1982-83, Birkhäuser, 1984.

[6] I. Satake, Algebraic structures of symmetrics domains. Pub. Math. Soc. Japan 14, Shoten Pub. and Princeton University Press, 1980.

[7] H. ShIGA AND J. Wolfart, Criteria for complex multiplication and transcendence properties of automorphic function. J. Reine Angew. Math 463 (1995), 1-25.

[8] G. SHIMURA, On analytic families of polarized abelian varieties and automorphic functions. Ann. Math. 78 (1963), 149-192.

[9] G. SHIMURA, Moduli and fibre systems of abelian varieties. Ann. of Math. 83 (1966), 294338.

[10] C.L. SiegeL, Lectures on Riemann matrices. Tata Institute, Bombay 1963.

[11] G. VAN DER GEER, Hilbert modular surfaces. Springer-Verlag, 1980.

[12] G. Wüstholz, Algebraic groups, Hodge theory, and Transcendence. Proc. ICM Berkeley 1 (1986), 476-483.

Geoffroy DEROME

Université des Sciences et Technologies

Bâtiment M2

59655 Villeneuve d'Ascq, France

E-mail : deromelagat.univ-lille1.fr 